SAND 99-0926

Unlimited Release

Printed August 1999

\title{
Photonic Band Gap Structures as a Gateway to Nano-Photonics
}

\author{
SY Lin, IJ Fritz \\ Photonic Research Department \\ V. Hietala, \\ Advanced Devices and Applications \\ JR Wendt, GA Vawter, G Hammons, JF Klem \\ Semiconductor Materials and Processes \\ SR Kurtz, \\ Advanced Semiconductor Technology \\ PL Gourley, \\ Nanostructure and Semiconductor Physics \\ SK Lyo, ED Jones. \\ Semiconductor Material and Device Science \\ Sandia National Laboratories \\ P.O. Box 5800 \\ Albuquerque, NM 87185-0603
}

\begin{abstract}
This report represents the completion of a three-year Laboratory-DirectedResearch and Development (LDRD) program to develop next generation photonic and optoelectronic devices based on a new material concept, namely the photonic band gap, or photonic crystal, materials. The work has focused on demonstration of novel devices that will pave the way to miniature photonic devices that is not only small in size but also integratable into one chip. Our effort has laid the ground work for high-quality bandpass filter for communication applications, highly dispersive prism for military and commercial applications as well as high efficient semiconductor lasers.
\end{abstract}




\section{Contents}

1. Introduction

2. High-Q Resonant Cavity Built from a Two-Dimensional Photonic Crystal.

3. Highly Dispersive Photonic Band Gap Prism.

4. Photonic Band Gap Edge-Emitting-Semiconductor-Lasers

5. Realization of Silicon Three-Dimensional Photonic Crystal

6. Experimental Demonstration of Guiding and Bending of Electromagnetic Waves in a TwoDimensional Photonic Crystal.

7. Conclusion.

8. References 


\section{DISCLAIMER}

This report was prepared as an account of work sponsored by an agency of the United States Government. Neither the United States Government nor any agency thereof, nor any of their employees, make any warranty, express or implied, or assumes any legal liability or responsibility for the accuracy, completeness, or usefulness of any information, apparatus, product, or process disclosed, or represents that its use would not infringe privately owned rights. Reference herein to any specific commercial product, process, or service by trade name, trademark, manufacturer, or otherwise does not necessarily constitute or imply its endorsement, recommendation, or favoring by the United States Government or any agency thereof. The views and opinions of authors expressed herein do not necessarily state or reflect those of the United States Government or any agency thereof. 


\section{DISCLAIMER}

Portions of this document may be illegible in electronic image products. Images are produced from the best available original document. 


\section{Introduction}

This LDRD project explored the fundamental physics of a new class of photonic materials, photonic bandgap structures (PBG), and examine its unique properties for the design and inplementation of photonic devices on a nano-meter length scale for the control and confinement of light. The low loss, highly reflective and quantum interference nature of a PBG material makes it one of the most promising candidates for realizing an extremely high-Q resonant cavity, $>10,000$, for optoelectronic applications and for the exploration of novel photonic physics, such as photonic localization, tunneling and modification of spontaneous emission rate. Moreover, the photonic bandgap concept affords us with a new opportunity to design and tailor photonic properties in very much the same way we manipulate, or bandgap engineer, electronic properties through modern epitaxy.

\section{High-Q Resonant Cavity Built from a Two-Dimensional Photonic Crystal}

We have tested a series of high-Q photonic bandgap (PBG) resonant cavities in the mmwave regime and achieved a cavity- $Q$ of 23,000 , to our knowledge, the highest value reported among all PBG-cavities. We have also systematically varied the size and reflectivity of such cavities to study their effect on cavity properties such as cavity modal frequency, linewidth and cavity Q-value. We show that the resonant frequencies can be tuned throughout the PBG regime and its linewidth (or equivalently Q-value) be varied over two order of magnitude (i.e. a $Q$ value from 200 to 23,000, see Fig.1(b)). Fig.1(a) shows the transmission spectrum of EM-waves through a PBG resonant cavity structure plotted as a function of frequency (f) from $70 \mathrm{GHz}$ to $110 \mathrm{GHz}$. The sharp peak, at $\mathrm{f}=85.5$ $\mathrm{GHz}$ corresponds to the resonant transmission of $\mathrm{EM}$ waves through the cavity mode. The observed cavity-Q of 23,000 is the highest ever been reported in any two- and threedimensional photonic band gap resonant cavities.

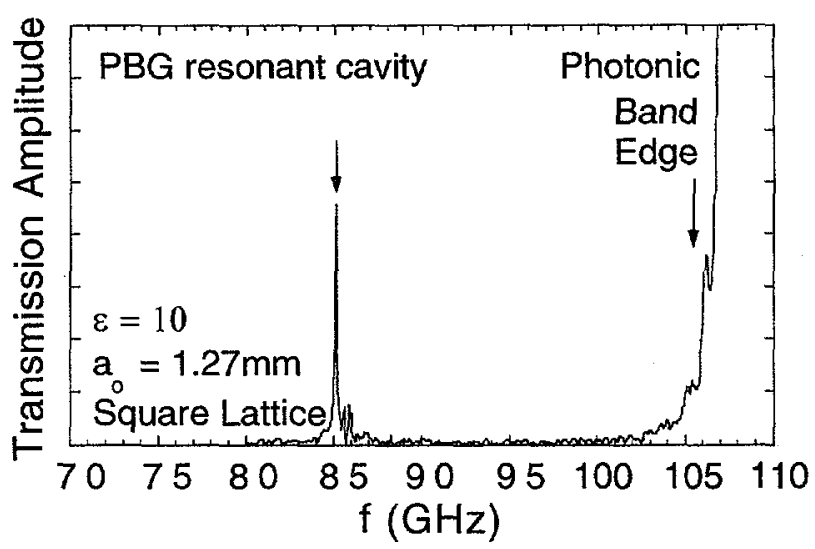

Fig.1(a) 


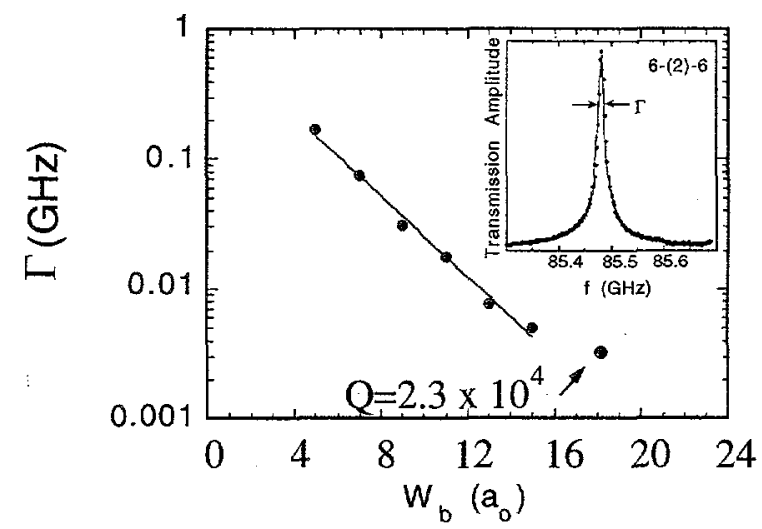

Fig.1(b)

\section{Highly Dispersive Photonic Band Gap Prism}

A photonic band gap (PBG) prism uses the highly non-linear dispersion of a PBG material near its band edge for dispersing light. A schematic of the top view of a twodimensional(2D) triangular PBG material is shown in Fig.2(a). By arranging the 2D array into a triangular shape, a 60 -degree prism is formed. The diameter, $\mathrm{d}$, and the lattice constant, a, are designed to yield the largest photonic band gap and therefore the strongest non-linear dispersion. A schematic plot of the effective index-dispersion for such a PBG-prism in shown in Fig.2(c), indicating a large index variation as frequency, $f$, is near the band edge. An experimentally determined index-dispersion is shown in Fig.2(d).

An experimental data showing the prism action is shown in Fig.3. Indeed, with the pbg prism, electromagnetic waves are well dispersed into an angle of 46.5 degree. The light is well directed as at 51.5-degree its intensity is dropped to less that $50 \%$ as compared to that measured at 46.5 degree.

An index variation of $20-30 \%$ in a well designed PBG-material is to be compared to the typical prism material's $\sim 1 \%$ index variation. With such a large index variation, a PBGprism can serve as a strong optical diepersive element. Although index variation of a crystalline material can be quite large near fundamental electronic band gap, yet, at such frequencies, the material suffers from serious absorption loss. Our PBG-material, on the other hand, is made of low loss dielectric material. Its photonic bandgap may be carefully chosen such that it is away from material's electronic bandgap and therefore minimize absorption loss. 


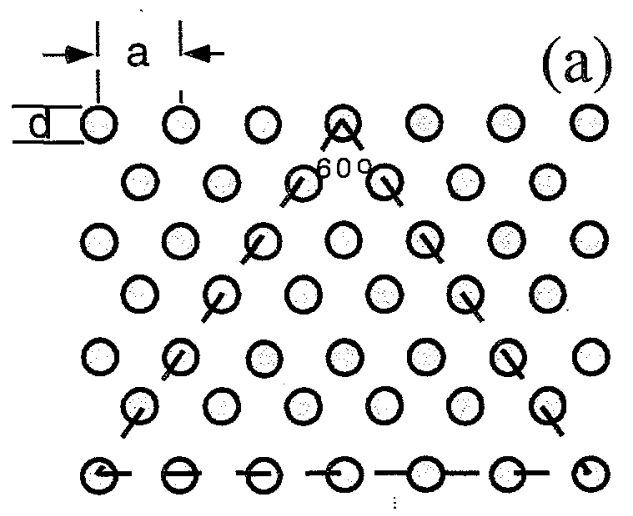

(b)

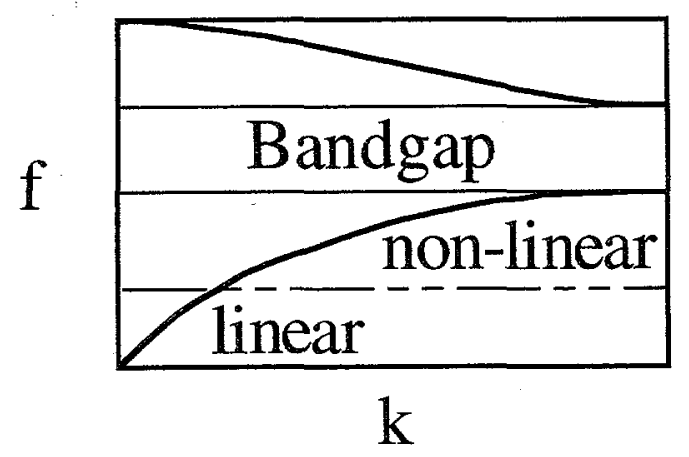

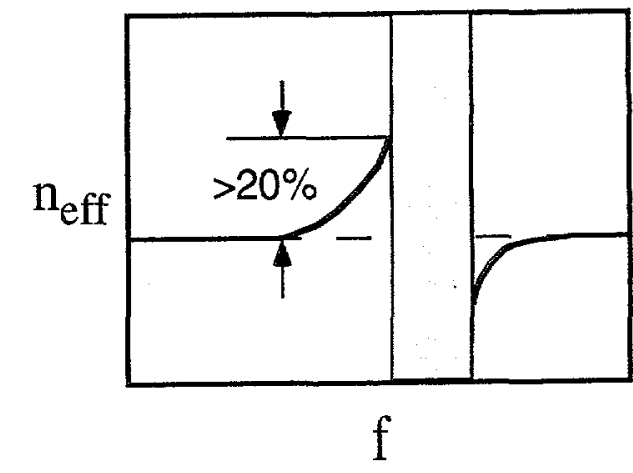

(c

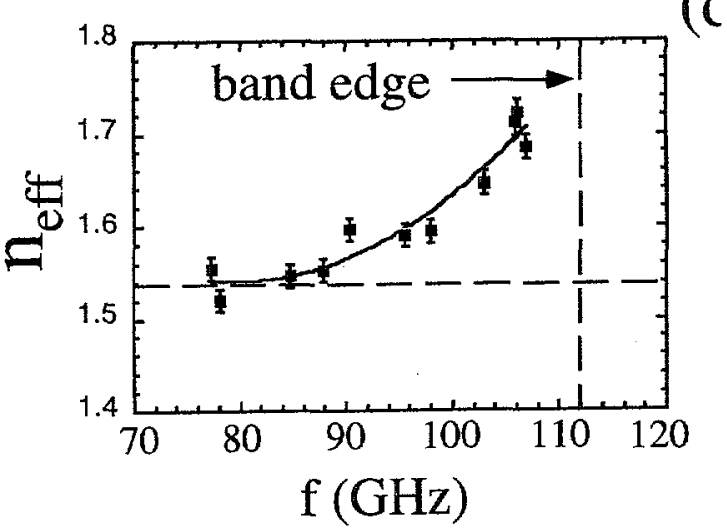

Fig.2 (a), (b), (c) and (d)

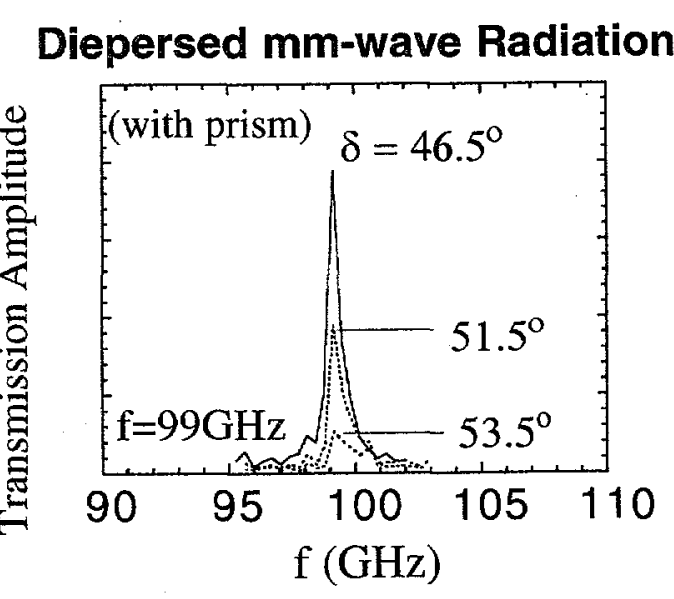

Fig.3 
We list three potential applications of a PBG-prism. The first one is a tunable and steerable tera-Hertz radiation source, see Fig.4(a). By combining and integrating an ultrafast optoelectronic switch (such as the Austin switch) with a PBG-prism, a compact tera-Hertz radiation souce is readily available. Another application is to use a prism to wavelength-selectively seed an optical amplifier. The seeding laser, the amplifier and the prism may all be integrated in one chip.

The third applications is in telecommunication, see fig.4 (b). In this case, a PBG-prism is used to dispersed light from an optical fiber. At a wavelength of $\lambda=1.5 \mu \mathrm{m}$, a prism of $50 \times 50 \mu \mathrm{m}^{2}$ and an area of $600 \mu \mathrm{m}$ is sufficient to dieperse 32 channel of optical signals. The prism and the output waveguides may be inter-connected with a planar waveguide. As a result, the device is not only compact but also planar integratable.

(a)

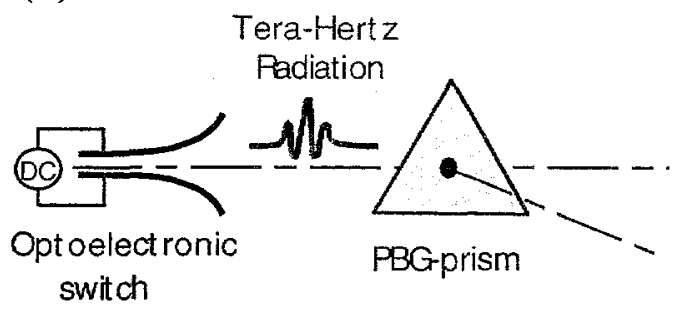

(b) optical fiber

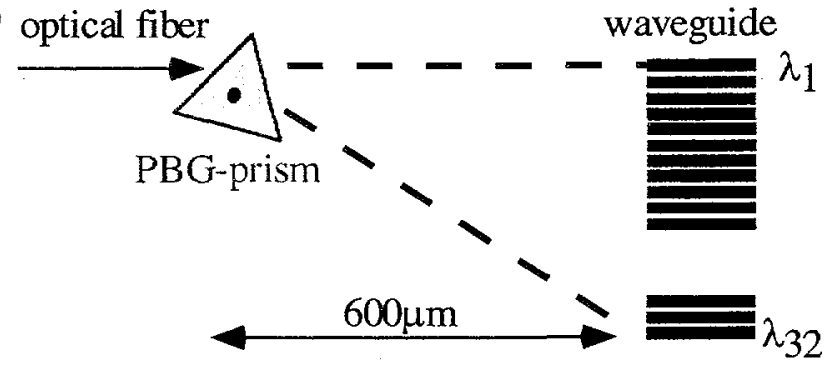

Fig.4(a) and (b)

\section{Photonic Band Gap Edge-Emitting-Semiconductor-Lasers}

A PBG-laser is a unique edge-emitting-laser that uses PBG materials as reflecting mirrors, rather than the conventional cleaved facets. Such a laser offers many advantages. The mirror reflectivity is adjustable and can be as high as $100 \%$. The mirror orientation is defined by lithography and thus can be aligned along any direction. In comparison, the conventional edge-emitting-laser is defined by cleaved facets along only major crystal orientations. The PBG-laser may also be placed anywhere on a wafer and its cavity length can be as short as sub-micron. With such a short cavity, single mode laser emission becomes possible. A schematic of a PBG-laser in shown in Fig.5.

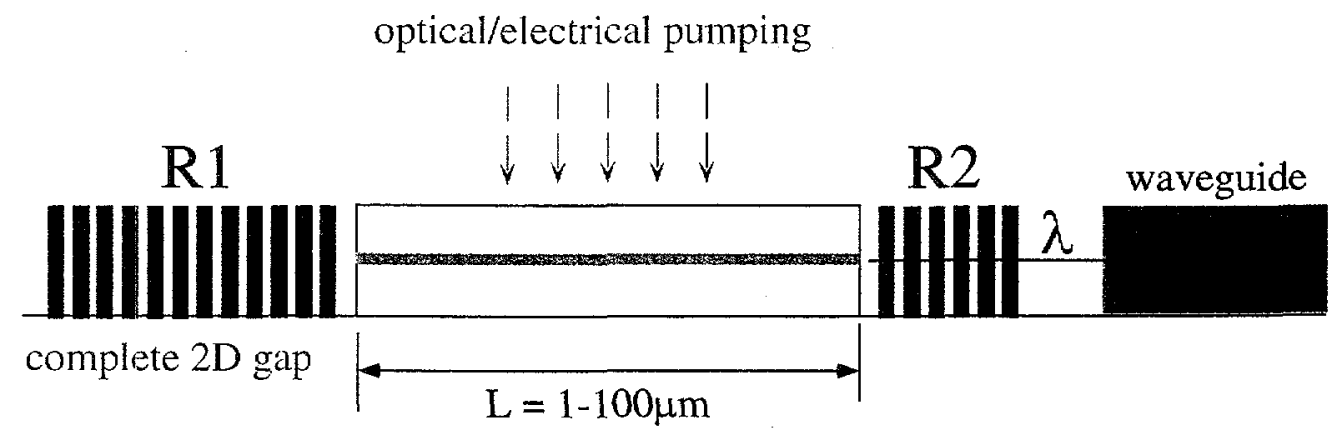

Fig.5 
A top and side view of a SEM image of a fabricated PBG-laser is shown in Fig.6(a) and 6(b) respectively. The $2 \mathrm{D}$ honeycomb PBG-structure constitutes the end mirror. It has a complete photonic band gap centered at $\lambda=920 \mathrm{~nm}$ and a bandwidth of $90 \mathrm{~nm}$. The square hole on the middle of the laser stripe is a contact window with $400 \mathrm{~nm}$ of Ti/Pt/Au. The $2 \mathrm{D}$ post has a diameter of $250 \mathrm{~nm}$ and a height of $2.0 \mu \mathrm{m}$. The structure is produced by the combination of electron-beam-lithography and reactive-ion-beam-etching technique.
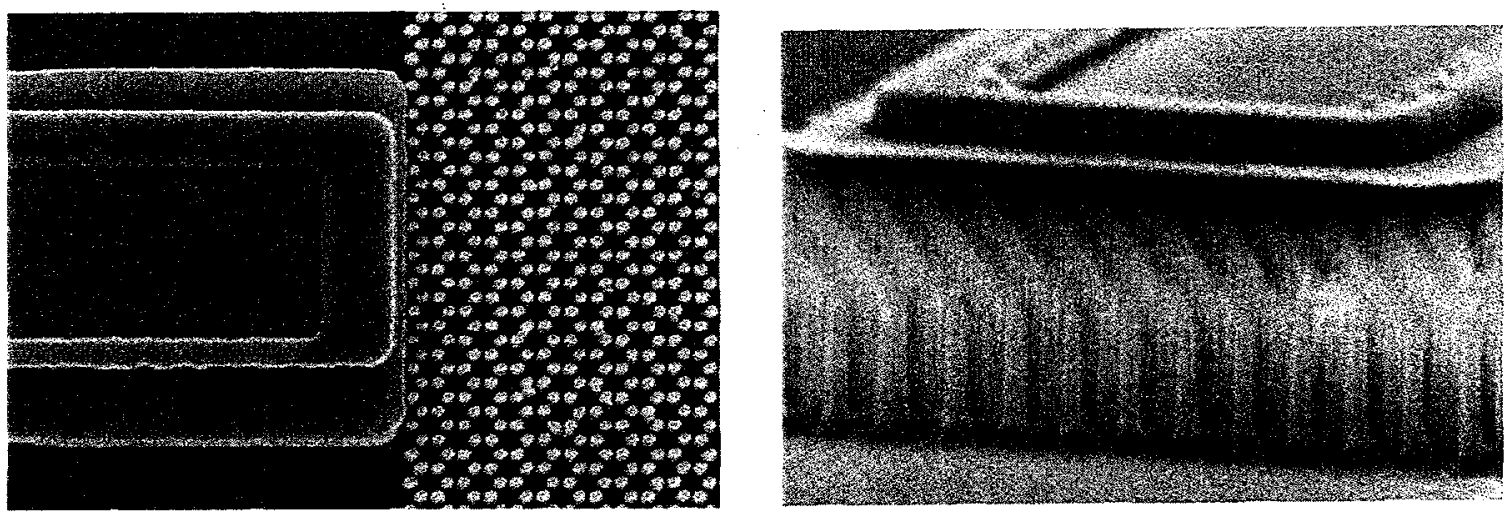

Fig.6(a) and (b)

A reflectance measurement of the 2D honeycomb PBG-mirror was carried out using a white light generated by an OPA laser system. A schematic of the measurement setup is shown in fig.7(a). The normalized data shown in Fig.7(b) is obtain by taking the ratio of (R-Ro)/Ro, where $\mathrm{R}$ is the reflectance from $2 \mathrm{D}$ posts and Ro a reference spectrum from substrate. The normalization procedure is necessary since the laser beam spot is larger than the post height and unwanted reflectance from sample substrate must be subtracted. A clear reflectance peak is seen at $\lambda \sim 940 \mathrm{~nm}$ consistent with the existence of a photonic band gap of approximately $40-60 \mathrm{~nm}$ bandwidth.

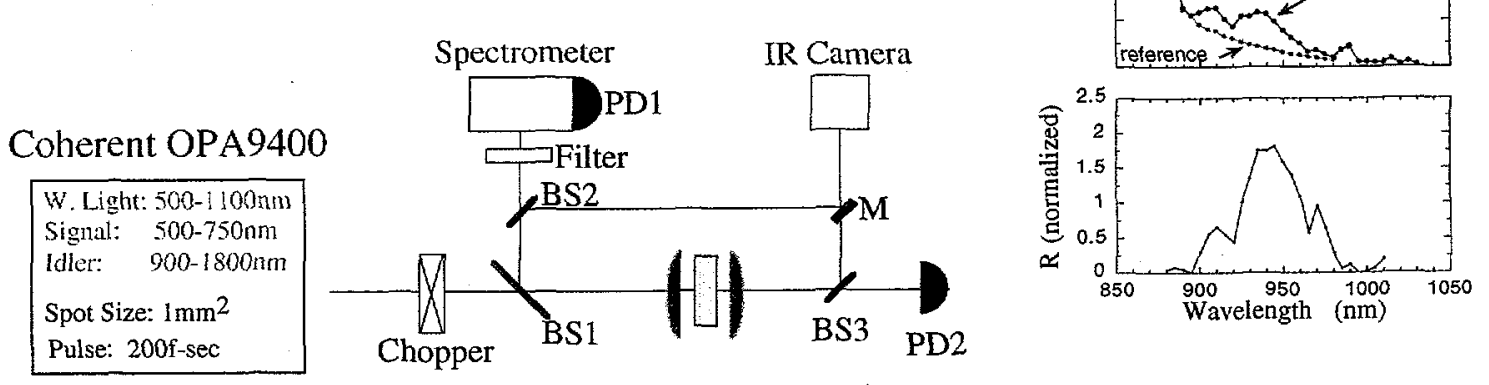

Fig.7(a) and (b) 
The light-current (L-I) curve and the lasing spectrum of a PBG-laser taken at room temperature is shown in Fig.8(a) and (b). The laser cavity is defined by a cleaved-facet on one end of he laser stripe and an etched facet coupled to the photonic lattice on the other end. The laser emits light at $\lambda=921 \mathrm{~nm}$ which falls in the bandgap regime, suggesting that the $2 \mathrm{D}$ honeycomb PBG-mirror may be responsible for the lasing. The lasing threshold is $125 \mathrm{~mA}$ for a sample length of $300 \mu \mathrm{m}$.
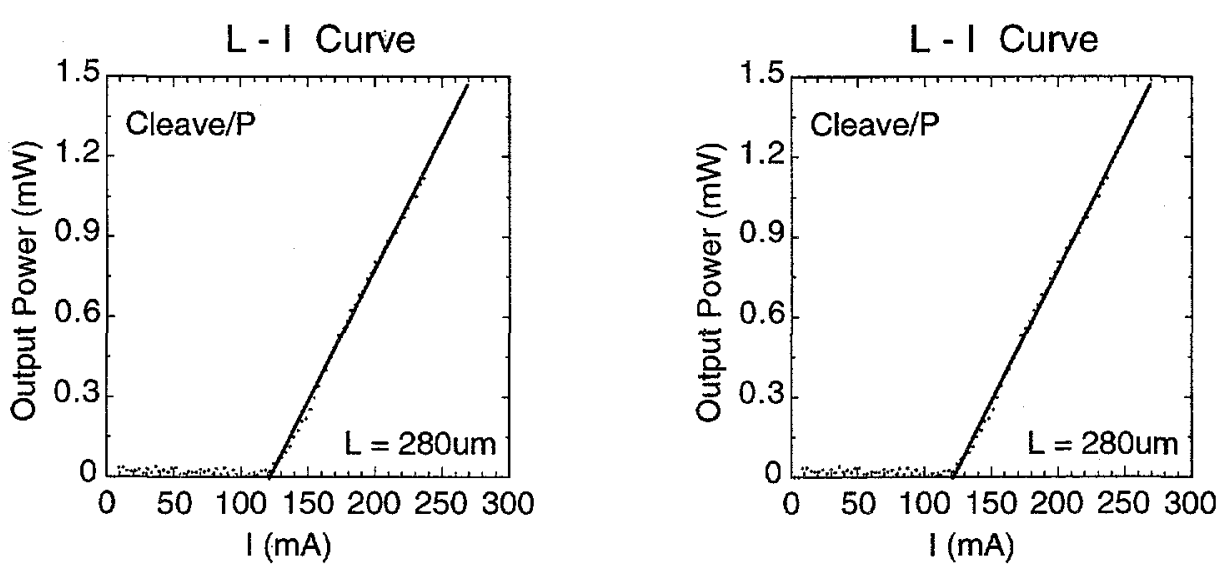

Fig. 8(a) and (b)

Given the expected high reflectance of a 2D photonic crystal, our lasing threshold results are certainly far from ideal. There are several causes: (1) the etched facet is not perfectly straight which will contribute to scattering loss at the laser-photonic crystal interface; (2) since a $2 \mathrm{D}$ photonic crystal is not designed to confine light along the post direction, some part of the lasing mode may propagate beneath the $2 \mathrm{D}$ posts and contributes to loss.

\section{Realization of Silicon Three-Dimensional Photonic Crystal}

Full three-dimensional control and confinement of light has important implications to quantum optics and quantum optical devices. Yet, the quest for 3D photonic crystals in the infrared and optical wavelength regime continue to challenge our state-of-art fabrication capabilities.

To create a 3D photonic crystal, we chose the so called layer-by-layer periodic structure for its design simplicity. An SEM image of a fabricated structure is shown in Fig.9(a). It consists of 5 layers of one-dimensional rods with a specific stacking sequence. The sample is fabricated on a 6-inch Silicon wafer. The one-dimensional rods consists of poly-silicon. The orientation of the axes are rotated by $90^{\circ}$ between adjacent layers. Between every other layers, the rods are shifted relative to each other by $1 / 2 \mathrm{~d}$. Here, $\mathrm{d}$ is the separation between adjacent rods. The resulting structure has a face-center-tetragonal lattice symmetry. 
The transmission spectrum of light propagating along the stacking direction of the 3D crystal is shown in Fig. 9 (b). At $\lambda=10-14 \mu \mathrm{m}$, a strong transmission dip is observed indicating the formation of photonic band gap. For this 7-layer structure, with a layer thickness of $\sim \lambda$, an attenuation of more than $98 \%$ is achieved. This is to be compared to the typical AlGaAs distributed-feedback-mirror, which uses 20-30 pairs (equivalent to $10-15 \lambda$ ) to achieve $98 \%$ reflectivity.

Such a $3 \mathrm{D}$ photonic crystal has many applications, ranginmg from thermal signal control to high precision temperature sensing. Our calculation also indicates that for an 8-layer structure, a 3D cavity can have a cavity-Q of $>5,000$. One can expect an enhanced silicon emission from such an high-Q cavity.
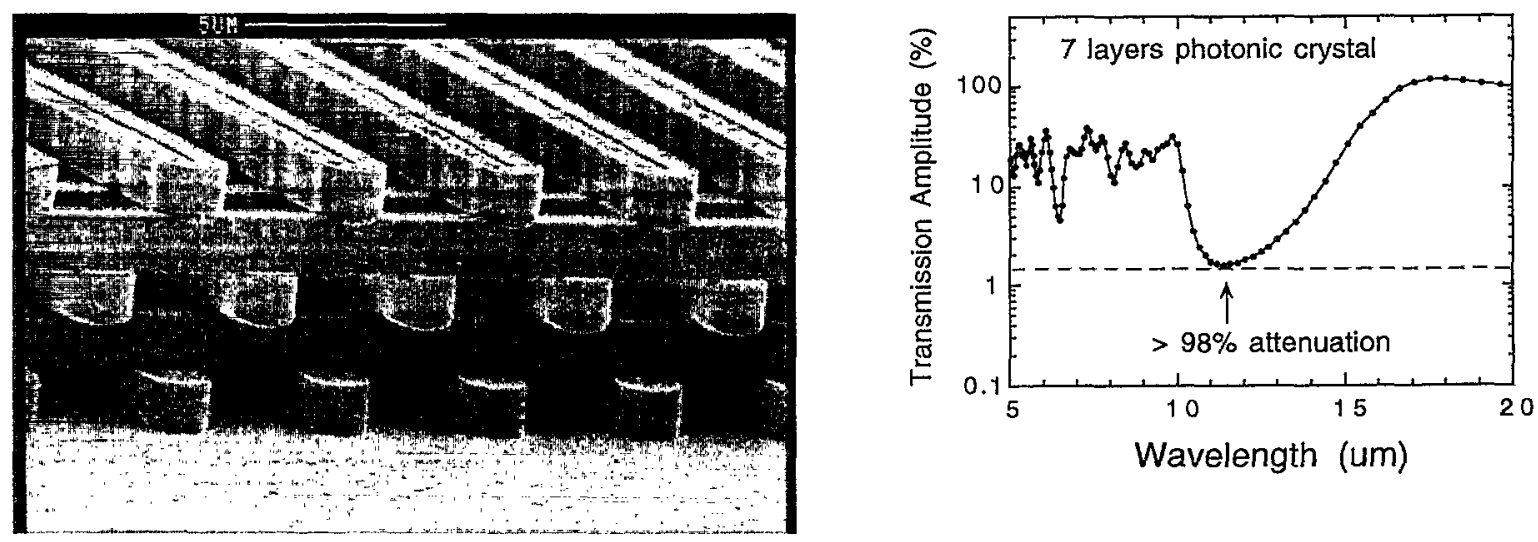

Fig. 9(a) and (b)

\section{Experimental Demonstration of Guiding and Bending of Electromagnetic Waves in a Two-Dimensional Photonic Crystal}

Guiding of light around a sharp corner with high efficiency is important for large scale all-optical circuit and optical computing applications. Recently, results of a computer simulation show a novel way to achieve this goal by using a photonic band gap waveguide. In this paper, we wish to report an experimental observation of a near $100 \%$ transmission through a photonic band gap 90-degree waveguide bend at certain specific frequencies, i.e. nodes in reflection. We further show that the position of the nodes can be model well with a simple one-dimensional scattering theory.

As we continue to drive for large integration of optical devices, the miniaturization of individual optical devices and the efficient routing of optical signals become important. 
Conventional dielectric waveguides can support straight guiding with high efficiency, yet are restricted by radiation loss to a moderate waveguide bending radius around a corner. Even for a high dielectric contrast guide, bending radius must exceed several wavelengths of the light to avoid large losses at the bending corner. Here, we report the first demonstration of waveguiding through a PBG line defect and more importantly the first observation of near perfect transmission around a sharp PBG waveguide bend.

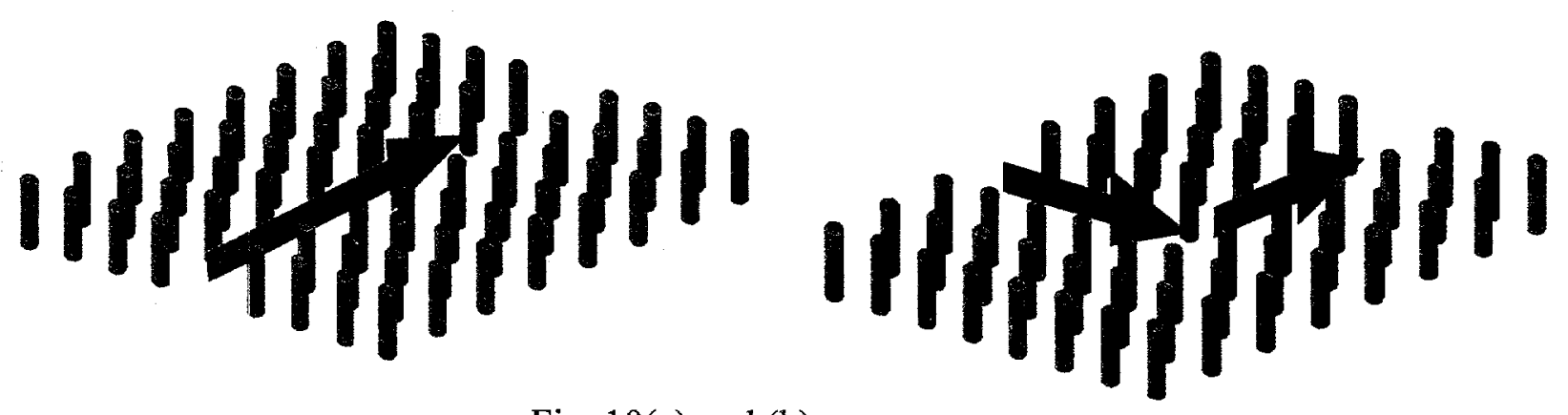

Fig. 10(a) and (b)
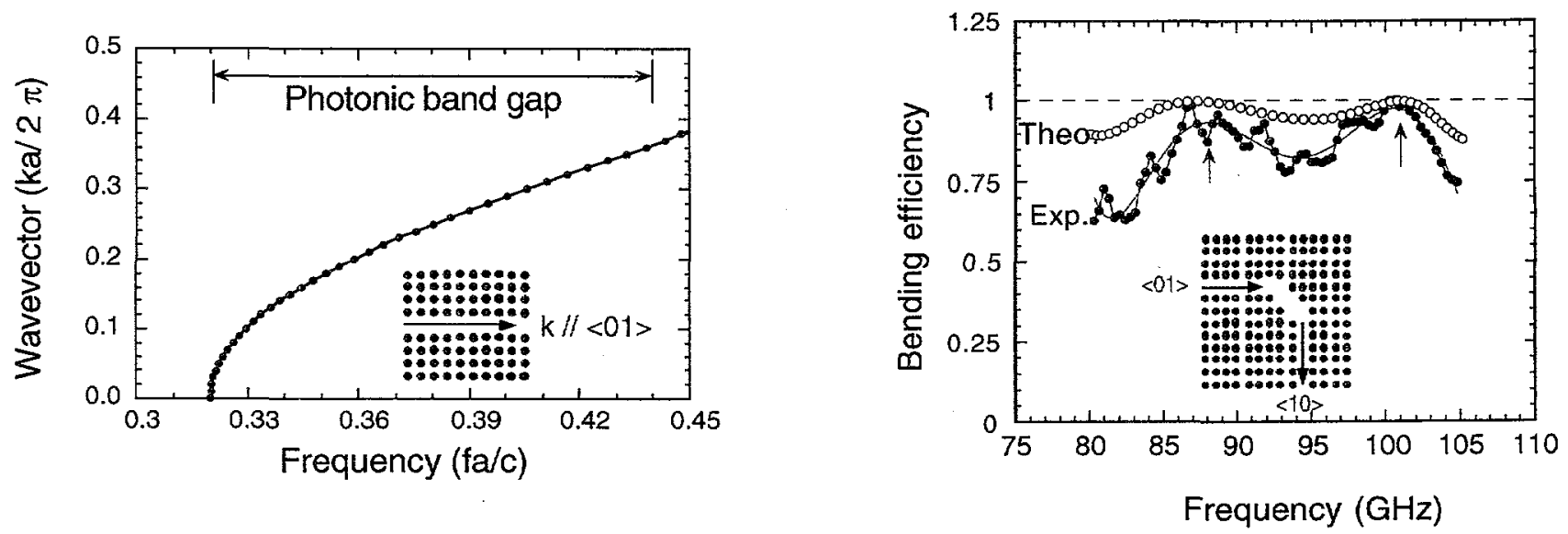

Fig.11 (a) and (b)

We chose to study a two-dimensional (2D) PBG structure of dielectric rods arranged in a square array with a lattice constant $a$. The refractive index of the rods is $n=3.0$ and their radius is $0.2 \mathrm{a}$. For such a 2D PBG material, the TM-gap extends from frequencies $\mathrm{f} 1=0.3 \mathrm{c} / \mathrm{a}$ to $\mathrm{f} 2=0.45 \mathrm{c} / \mathrm{a}$. A straight $\mathrm{PBG}$-waveguide is introduced by removing one row of rods, shown in Fig.10(a). The resulting TM-mode is localized perpendicular to the guiding direction $\mathrm{x}$ and at the same time experiences translational symmetry along the guide. Thus, we have created a 1D single guided TM mode inside the band gap. The degree of localization depends on the modal frequency and is the strongest at the mid- 
gap. Due to its translaional symmetry, the 1D PBG-waveguide can be described by a diepersion relationship, i.e. frequency vs wavevector, and is plotted in Fig.11(a). The modal frequency extends from $f=0.30 \mathrm{c} / \mathrm{a}$ at $\mathrm{G}$ point to the upper band edge $f=0.45 \mathrm{c} / \mathrm{a}$ at $\mathrm{k}=0.35$.

Having establishing that a PBG 1D-defect acts as a straight waveguide, we now study a 90 -degree waveguide bend. The bend is constructed by joining two straight waveguides at a 45-degree PBG-corner, shown in Fig.10(b). The straight waveguides are oriented along the $\langle 10\rangle$ and $<01\rangle$ crystal directions and the 45-degree corner section along the $<11>$ directionThe PBG-corner functions as a dielectric mirror. To test the device, the transmitter and receiver are again placed right next to the entrance and exit of the PBG bend. To obtain intrinsic bending efficiency of a PBG-bend, we need to compenstate for the frequency-dependent coupling efficiency. The following normalization scheme was used. Two transmission spectra were taken sequentially, one from a straight waveguide (T0) and the other from the bend (T1). By normalizing T1 to T0, we eliminate the effect due to input/output coupling and are left with the intrinsic bending response. The normalized transmission amplitude (T1/T0) is plotted in Fig.11(b). We observed a near $100 \%$ bending efficiency, nodes in reflection, at two specific frequencies, $\mathrm{f}=85 \mathrm{GHz}$ and $99 \mathrm{GHz}$. This measurement demonstrates the existance of nodes at which no back reflection is possible. It also confirms that no power can be radiated out of the bend since there are no extended modes into which the propagation mode can leak out.

In summary, we describe the first dispersion measurement of surface EM-Bloch-wave confined at the air/ photonic lattice interface. The measured dispersion agrees well with our theoretical prediction. We further determine its spatial extend into the air and find that it is of highly localized.

\section{Conclusion}

In conclusion, our "photonic band gap material research" LDRD has resulted in a very significant expansion of Sandia's nano-photonic technology capability. Photonic band gap approach to infrared military sensing application, to integrated photonic circuits applications based on silicon technology, and to optical communication applications such as ultrafast optical switches and high efficient lasers will certainly impact the whole photonic inductry in the years to come. 


\section{References}

1. SY Lin et al, "Photonic band gap quantum well and quantum dot structures: a high-Q resonant cavity ", Appl. Phys. Lett.,68, 3233 (1996).

2. SY Lin et al, "Photonic band gap high-Q resonant cavity: from mm-wave to optical regime ",SPIE proceeding, Vol. 2693, p.170, "Physics and simulation of optoelectronic devices IV" (1996).

3. SY Lin et al, "Highly dispersive photonic band-gap prism", Optics Letters 21, p.17711773 (1996).

4. SY Lin et al, "Three-dimensional photonic crystal operating at infrared wavelengths", Nature, vol 394, p. 251-253, July 17, 1998.

5. SY Lin et al, "Experimental demonstration of guiding and bending of light in a photonic crystal", Science, Vol 282, 274(1998). 
2 MS 0603 Shawn Lin (1712)

1 MS 0603 Joel Wendt (1711)

1 MS 0603 Ian Fritz (1712)

1 MS 0603 Alan Vawter (1711)

1 MS 0603 John Klem (1711)

1 MS 0603 Charles Sullivan (1713)

1 MS 0603 Mial Warren (1712)

1 MS 0603 Peter Esherick (1711)

1 MS 0603 Steve Kurtz (1713)

1 MS 0603 Tom Zipperian (1713)

1 MS 0603 Carol Ashby (1711)

1 MS 0874 Vince Hietala

1 MS 0874 Dave Palmer

1 MS 1056 Paul Gourley

1 MS 1056 Ken Lyo

1 MS 1056 Wil Gauster

1 MS $0601 \quad$ Eric Jones

1 MS 0601 Jeff Nelson

1 MS 9018 Central Technical Files (8940-2)

2 MS 0899 Technical Library (4916)

1 MS 0619 Review \& Approval Desk (00111)

1 MS 0161 Patent and Licensing Office (11500)

1 For DOE/OSTI

1 MS $0188 \quad$ LDRD Office (4001) 